\title{
Effects of recombinant human adenovirus type 5 combined with transarterial chemoembolization on postoperative metastasis and recurrence of hepatocellular carcinoma patients
}

\author{
Ke Wu, Nan You, Lu Zheng \\ Department of Hepatobiliary Surgery, The Second Affiliated Hospital of Army Medical University, Chongqing, China \\ Contributions: (I) Conception and design: L Zheng; (II) Administrative support: L Zheng; (III) Provision of study materials or patients: K Wu; (IV) \\ Collection and assembly of data: N You; (V) Data analysis and interpretation: K Wu; (VI) Manuscript writing: All authors; (VII) Final approval of \\ manuscript: All authors. \\ Correspondence to: Lu Zheng. Department of Hepatobiliary Surgery, The Second Affiliated Hospital of Army Medical University, 83 Xinqiao Main \\ Street, Shapingba District, Chongqing 400037, China. Email: xqyyzl1@163.com.
}

Background: Postoperative recurrence is currently the main factor affecting the long-term survival of
hepatocellular carcinoma (HCC) patients. The folinic acid, fluorouracil, and oxaliplatin (FOLFOX) regimen
with transarterial chemoembolization (TACE) is a commonly used postoperative chemotherapy strategy, but
its effect is still limited. The aim of this study was to analyze the effects of recombinant human adenovirus
type 5 (rhAd5) combined with TACE on postoperative metastasis and recurrence of HCC.
Methods: Patients with HCC undergoing surgical treatment were collected and divided into the rhAd5
group and control group according to whether rhAd5 was performed. The rhAd5 group was combined
with rhAd5 treatment based on TACE. The recurrence and metastasis rates of the two participant groups
were compared. The changes of liver function, kidney function, blood routine, and adverse reactions during
treatment were analyzed.

Results: The basic data of the two groups were not significantly different $(\mathrm{P}>0.05)$. The recurrence and metastasis rates of rhAd5 group participants were significantly lower than those of the control group $(\mathrm{P}<0.05)$. There was no significant difference in the incidence of adverse reactions between the rhAd5 group and control group $(\mathrm{P}>0.05)$. There were no significant differences in the incidences of adverse reactions between the rhAd5 group and control group $(\mathrm{P}>0.05)$.

Conclusions: The combination of FOLFOX and rhAd5 after surgery can significantly inhibit the occurrence of metastasis and recurrence of HCC patients, improve progression free survival, and has certain safety.

Keywords: Hepatocellular carcinoma (HCC); oncolytic virus; recombinant human adenovirus type 5 (rhAd5)

Submitted Sep 24, 2021. Accepted for publication Dec 16, 2021.

doi: 10.21037/jgo-21-792

View this article at: https://dx.doi.org/10.21037/jgo-21-792

\section{Introduction}

A study of 185 countries showed that the number of new hepatocellular carcinoma (HCC) patients in 2018 was 841,080 , accounting for $4.7 \%$ of all tumors. The number of deaths among HCC patients in 2018 was 782,685 , accounting for $8.2 \%$ of all deaths from tumors, ranking second overall (1). Presently, surgery is still the main method of treating primary $\mathrm{HCC}(2,3)$. However, due to the rich local blood vessels, HCC cells are highly metastatic $(4,5)$. Further, whether and how long the recurrence of HCC will occur after tumor resection is related to the number of tumor nodules, tumor size and the range of involvement. It has reported that the recurrence rate of HCC is as high as $80 \%$ five years after tumor resection (6). The poor prognosis 
of HCC patients is mainly due to the high frequency of advanced lesions, metastasis and tumor reformation in the diseased liver, that is, the so-called "field effect". After surgery, the use of chemotherapeutic drugs can effectively reduce the recurrence and metastasis of HCC (7-9). Folinic acid, fluorouracil, and oxaliplatin (FOLFOX) is an effective chemotherapeutic regimen after surgery (10). Unfortunately, many HCC patients receive little benefit from transarterial chemoembolization (TACE) using FOLFOX.

Oncolytic viruses are genetically engineered viruses that can specifically target tumor cells (11). In normal cells, oncolytic viruses are cleared by the body's immune system. In tumor cells, due to immune escape, oncolytic viruses can replicate and eventually traffic to the tumor environment and cause tumor kill (12). The clinical trial of oncolytic viruses as a single drug is largely safe and proves its minimal toxicity (13). Oncolytic virotherapies have proven themselves to be potentially powerful tools to fight malignancy. One potential limitation of oncolytic viruses, which is often debated, is the rapid production of antiviral antibody response about a week after injection. The true potential of oncolytic viruses may be realized through their combination with chemotherapeutic drugs, which may help to overcome some obstacles that limit the efficacy of oncolytic viruses in the tumor microenvironment. Adenovirus is a type of oncolytic virus, and one clinical study has shown that adenovirus-p53 combined chemotherapy has better effects on solid tumors (14).

Recombinant human adenovirus type $5(\operatorname{rhAd} 5)$ is a kind of modified oncolytic virus. Genetic engineering technology is used to delete the gene fragments of $\mathrm{E} 1$ and $\mathrm{E} 3$ regions that related to virus replication $(15,16)$. It not only could improve the safety of medication, but also allow rhAd5 to selectively replicate in tumor cells. The method of applying rhAd5 is usually intratumoral injection with TACE. TACE could send rhAd5 directly into vessels in tumor and prevent rhAd5 break by host immune system, thereby increasing the available rhAd5 concentration and reducing its impact on the body. Dong et al. (17) and Lin et al. (18) have reported that that rhAd5 combined with TACE was a safe, effective therapeutic strategy that can prolong the progression-free survival (PFS) and overall survival (OS) time of patients with unresectable HCC. However, there is no research about the effects of rhAd5 on the recurrence and metastasis of HCC patients undergoing surgical treatment.

The purpose of this study was to evaluate the effects of rhAd5 combined with FOLFOX treatment on postoperative metastasis and recurrence of HCC patients, and to evaluate its safety.

We present the following article in accordance with the STROBE reporting checklist (available at https://dx.doi. org/10.21037/jgo-21-792).

\section{Methods}

\section{Study population}

This is a retrospective study, a total of 64 patients with HCC who underwent surgery at The Second Affiliated Hospital of Army Medical University from January 2015 to January 2016 were recruited. The patients were divided into the rhAd5 group and control group according to whether received rhAd5 injection. All participants had provided their informed consent. This study was approved by the Ethics Review Committee of The Second Affiliated Hospital of Army Medical University (No.: CQ-012-201434532) and was conducted in accordance with the Declaration of Helsinki (as revised in 2013).

\section{Enrollment criteria}

All participants were diagnosed with HCC based on imaging and pathological tests. The inclusion criteria were as follows: (I) >18 years old, regardless of gender; (II) met the criteria for surgical treatment; (III) received postoperative TACE and rhAd5 treatment. The exclusion criteria were as follows: (I) had received chemotherapy, targeted therapy, or radiation therapy before surgery; (II) with other primary tumors; (III) with hematological diseases and immune system diseases; (IV) with other liver diseases.

\section{Treatment of TACE and rhAd5}

The participants of control group were treated with TACE 1-3 months after surgery. According to the results of proper hepatic arteriography, the catheter was inserted into the area of the artery supplying the tumor. Firstly, deoxyfluridine $1,000 \mathrm{mg}$ was given intra-arterial infusion chemotherapy for 15 mins. Then, oxaliplatin $50 \mathrm{mg}$ and super-liquid lipiodol were applied for TACE treatment. After TACE treatment, the tip of the catheter was placed in the proper hepatic artery, and oxaliplatin $100 \mathrm{mg}(2 \mathrm{~h})$ and deoxyfluridine $200 \mathrm{mg}$ $(44 \mathrm{~h})$ were sequentially pumped through the catheter artery before intravenous infusion of Leucovorin $300 \mathrm{mg}$. For the rhAd5 group, rhAd5 (Oncorine, Sunway Biotech, Shanghai, China) was added at the same time as TACE, the dose was 
$1.5 \times 10^{12} \mathrm{vp} /$ time. The treatment was repeated every 4 weeks for 4 consecutive times.

\section{Indicators for comparison}

Before treatment, the general information of all participants was collected, including Child-Pugh classification, American Society of Anesthesiologists (ASA) classification, tumor size, liver function, renal function, blood routine, hepatitis B surface antigen ( $\mathrm{HBsAg}$ ), hepatitis B virus DNA (HBV-DNA), and hepatitis C virus (HCV) infection status. The above laboratory indicators were monitored every week after treatment. The progress, recurrence, and metastasis of HCC in the two groups of participants were followed up. The judgment criteria for recurrence diagnosis were as follows: B-ultrasound, computed tomography (CT), magnetic resonance imaging (MRI), and the identification of liver-occupying lesions via other imaging examinations, consistent with the characteristics of primary HCC, postoperative serum alpha fetoprotein (AFP) rose to more than $200 \mathrm{~g} / \mathrm{L}$, to the exclusion of active liver disease. PFS was the interval between the time of surgical resection and relapse. All hematology tests were performed in the laboratory. The peripheral venous blood samples were sent for inspection immediately after collection, and the test results were recorded in the computer.

\section{Statistical analysis}

The software SPSS 19.0 (IBM Corp., Armonk, NY, USA) was used to execute statistical analyses with Student's $t$-tests for 2 groups. The count data was used $t$-test.

\section{Results}

\section{Clinical characteristics of patients}

Among the 64 participants, there were 47 males and 17 females, with ages ranging from 19 to 82 years, and the median age was 53 years. In the control group, there were 22 males and 10 females $(n=32)$ aged 19-80 years old, and the median age was 52 years. In the rhAd5 group $(n=32)$, there were 25 males and 7 females, aged 22-82 years, and the median age was 53 years. As Table 1 shown, the clinical characteristics of the two participant groups were compared. There was no significant difference in terms of age, gender, infected with hepatitis B virus (HBV), tumor sizes, the differentiations of HCC, blood routine, liver function and kidney function indexes between two groups (all $\mathrm{P}>0.05$, Table 1). Pathological analysis was performed by immunohistochemical (IHC) staining (Figure 1A,1B).

\section{Treatment effectiveness analysis}

All participants achieved complete resection after treatment. The deadline for follow-up was December 2019. In the control group, there were 16 cases $(50.00 \%)$ of intrahepatic recurrence, including two cases $(6.25 \%)$ of brain metastases and 14 cases $(43.75 \%)$ of lung metastases. At the follow-up date, a total of 20 patients $(62.50 \%)$ had died.

In the rhAd5 group, there were 11 cases $(34.38 \%)$ of intrahepatic recurrence, one case $(3.12 \%)$ of brain metastasis, and ten cases (31.25\%) of lung metastasis. At the follow- up date, a total of 12 participants (37.50\%) died. The average OS of the rhAd5 group and control group were $46.04 \pm 18.11$ and $34.84 \pm 19.27$ months, respectively. The average PFS of the rhAd5 group and control group were $37.19 \pm 18.57$ and $21.43 \pm 11.14$ months, respectively. The average OS and PFS of the rhAd5 group were longer than those of the control group $(t=2.419, \mathrm{P}=0.022 ; t=4.330$, $\mathrm{P}<0.001$, respectively). The OS and PFS rates of the rhAd5 group were significantly higher than those of the control group (Figure 2A,2B).

\section{Adverse reactions}

There was no liver, kidney, or cardiotoxicity above grade 3 in both groups. The most common complications were fatigue, nausea and vomiting, diarrhea, and bone marrow suppression. There was no significant difference in the incidence of adverse reactions between the control group and rhAd5 group $(\mathrm{P}>0.05)$. The symptoms were mild and no additional treatment was needed. The detail results were shown in Figures 3,4.

\section{Laboratory indicators}

The liver function index, kidney function index, and blood routine of the two groups were compared, and the peak value of each participant index fluctuation were recorded. The results showed that there was no statistically significant difference between the two groups (all $\mathrm{P}>0.05$, Table 2).

\section{Discussion}

The liver microenvironment consists of a complex network 
Table 1 General characteristics of unresectable HCC patients in the two groups

\begin{tabular}{|c|c|c|c|c|}
\hline Characteristics & $\begin{array}{l}\text { Total } \\
(n=64)\end{array}$ & $\begin{array}{l}\text { rhAd5 group } \\
\qquad(n=32)\end{array}$ & $\begin{array}{l}\text { Control } \\
(n=32)\end{array}$ & $P$ value \\
\hline Age & & & & 0.794 \\
\hline$\leq 60$ & 41 & 21 & 20 & \\
\hline$>60$ & 23 & 11 & 12 & \\
\hline Gender & & & & 0.396 \\
\hline Male & 47 & 25 & 22 & \\
\hline Female & 17 & 7 & 10 & \\
\hline $\mathrm{HbsAg}$ & & & & 0.564 \\
\hline Positive & 48 & 25 & 23 & \\
\hline Negative & 16 & 7 & 9 & \\
\hline HBV-DNA & & & & 0.611 \\
\hline$<100$ & 26 & 12 & 14 & \\
\hline$\geq 100$ & 38 & 20 & 18 & \\
\hline ALT (U/L) & & & & 0.448 \\
\hline$<40$ & 37 & 20 & 17 & \\
\hline$\geq 40$ & 27 & 12 & 15 & \\
\hline AST (U/L) & & & & 0.798 \\
\hline$<45$ & 39 & 20 & 19 & \\
\hline$\geq 45$ & 25 & 12 & 13 & \\
\hline TBIL (mmol/L) & & & & 0.768 \\
\hline$<20$ & 49 & 24 & 25 & \\
\hline$\geq 20$ & 15 & 8 & 7 & \\
\hline DBIL (mmol/L) & & & & 0.162 \\
\hline$<3$ & 59 & 31 & 28 & \\
\hline$\geq 3$ & 5 & 1 & 4 & \\
\hline ALB (U/L) & & & & 0.790 \\
\hline$<45$ & 43 & 22 & 21 & \\
\hline$\geq 45$ & 21 & 10 & 11 & \\
\hline WBC $\left(\times 10^{9} / \mathrm{L}\right)$ & & & & 0.313 \\
\hline$<10$ & 63 & 32 & 31 & \\
\hline$\geq 10$ & 1 & 0 & 1 & \\
\hline PLT (×10\% $/ \mathrm{L})$ & & & & 0.719 \\
\hline$<100$ & 9 & 4 & 5 & \\
\hline$\geq 100$ & 55 & 28 & 27 & \\
\hline
\end{tabular}

Table 1 (continued)
Table 1 (continued)

\begin{tabular}{|c|c|c|c|c|}
\hline Characteristics & $\begin{array}{l}\text { Total } \\
(n=64)\end{array}$ & $\begin{array}{l}\text { rhAd5 group } \\
\quad(n=32)\end{array}$ & $\begin{array}{l}\text { Control } \\
(n=32)\end{array}$ & $P$ value \\
\hline \multicolumn{4}{|l|}{ HB (g/L) } & 0.424 \\
\hline$<150$ & 43 & 23 & 20 & \\
\hline$\geq 150$ & 21 & 9 & 12 & \\
\hline \multicolumn{4}{|l|}{ PT (s) } & 0.528 \\
\hline$<11$ & 16 & 9 & 7 & \\
\hline$\geq 11 \&<13$ & 39 & 20 & 19 & \\
\hline$\geq 13$ & 9 & 3 & 6 & \\
\hline \multicolumn{4}{|l|}{ AFP (ng/mL) } & 0.614 \\
\hline$<25$ & 28 & 13 & 15 & \\
\hline$\geq 25$ & 36 & 19 & 17 & \\
\hline \multicolumn{4}{|c|}{ Creatinine $(\mu \mathrm{mol} / \mathrm{L})$} & 0.323 \\
\hline$<100$ & 57 & 30 & 27 & \\
\hline$\geq 100$ & 7 & 2 & 5 & \\
\hline \multicolumn{3}{|c|}{ Urea nitrogen (mmol/L) } & 0.32 & 0.32 \\
\hline$<7.5$ & 53 & 28 & 25 & \\
\hline$\geq 7.5$ & 11 & 4 & 7 & \\
\hline \multicolumn{4}{|l|}{ Tumor size (cm) } & 0.802 \\
\hline$<5$ & 35 & 18 & 17 & \\
\hline$\geq 5$ & 29 & 14 & 15 & \\
\hline \multicolumn{4}{|c|}{ Number of tumors } & 0.616 \\
\hline Single & 30 & 14 & 16 & \\
\hline Multiple & 34 & 18 & 16 & \\
\hline
\end{tabular}

HCC, hepatocellular carcinoma; HbsAg, hepatitis B surface antigen; HBV-DNA, hepatitis B virus DNA; ALT, alanine transaminase; AST, aspartate transaminase; TBIL, total bilirubin; DBIL, direct bilirubin; PLT, platelet; HB, hemoglobin; AFP, alpha fetoprotein.

of hepatocytes, stromal cells, inflammatory cells, and extracellular matrix (ECM). Surgery is the main treatment method for HCC, but the recurrence and metastasis rate of HCC is high (19). The main reason for post-surgical recurrence of HCC is malignant biological characteristics, such as incomplete resection or the presence of hidden lesions and some tumor cells enter the blood vessel and transfer to other sites for colonization $(5,20)$. Therefore, new alternative therapies are needed, such as the use of 

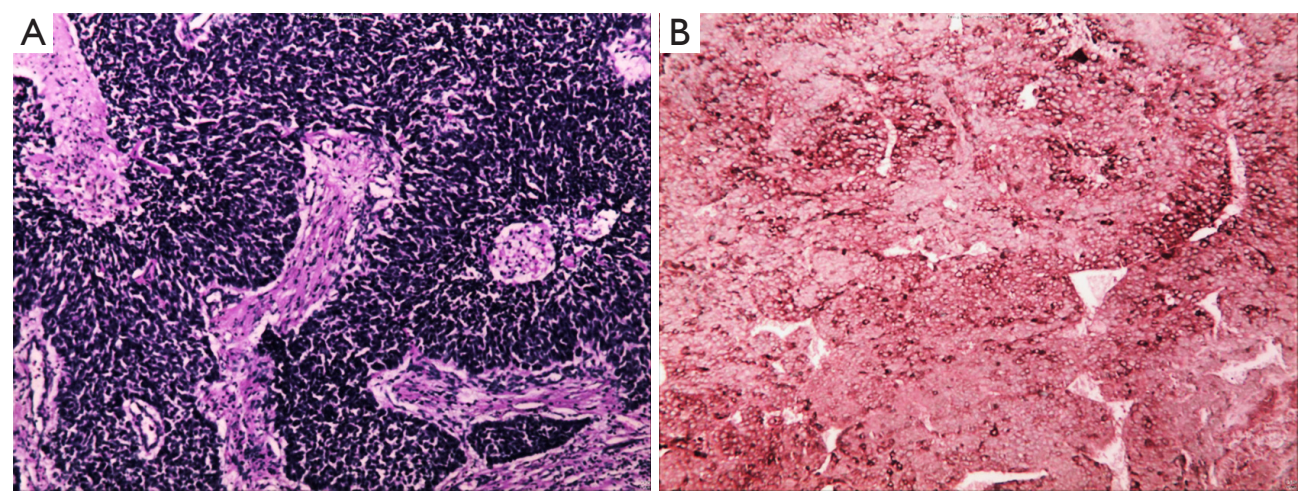

Figure 1 Results of pathology analysis. (A) Male, 56 years old, tumor size was $9.5 \mathrm{~cm} \times 4 \mathrm{~cm} \times 4 \mathrm{~cm}$, Ki67 hot spot was about $70 \%$. (B) Male, 73 years old, tumor size was $6 \mathrm{~cm} \times 5 \mathrm{~cm} \times 3 \mathrm{~cm}$, Ki67 hot spot was about $60 \%$. Hematoxylin-eosin (HE) staining; magnification, $\times 20$.

A

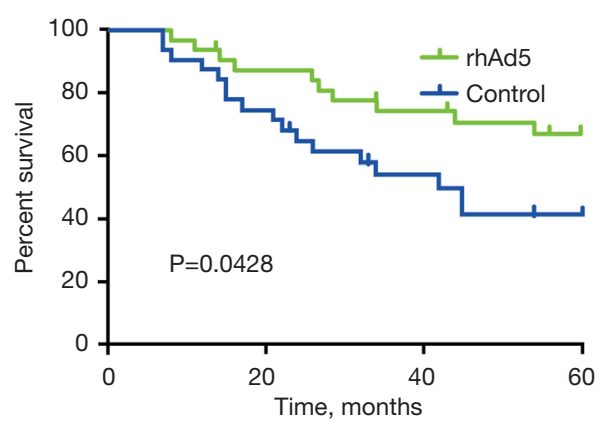

B

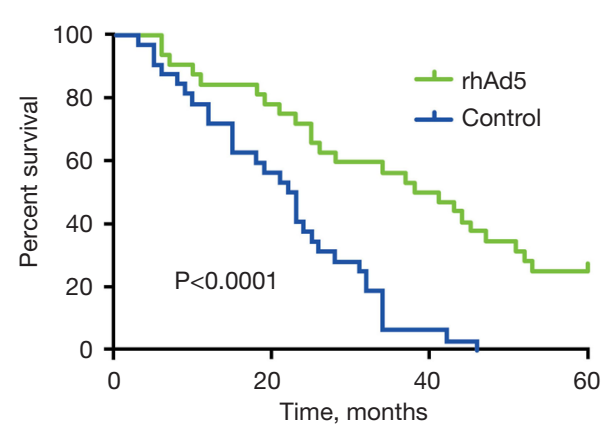

Figure 2 Comparison of OS and PFS between control group (TACE with FOLFOX) and rhAd5 group (TACE with FOLFOX + rhAd5). (A) Comparison of OS between control group and rhAd5 group. (B) Comparison of PFS between control group and rhAd5 group. OS, overall survival; PFS, progression free survival; TACE, transarterial chemoembolization; FOLFOX, folinic acid, fluorouracil, and oxaliplatin.

oncolytic viruses. Liver is one of the three most sensitive organs to hypoxia, it has been found that hypoxia is related to HCC metastasis, poor prognosis and radiotherapy resistance. As we learn more about the pathogenesis of liver disease, we can actually exploit the unique features of the local microenvironment to synergize with oncolytic viruses therapy, and thereby reduce the postoperative recurrence and metastasis of HCC.

Postoperative chemotherapy is important to eliminate residual lesions and inhibit metastasis. The TACE is a combination of platinum compounds, which can reduce tumor recurrence after surgery $(21,22)$. In this study, FOLFOX and TACE were used after surgery, and rhAd5 was used in combination with intervene. There was no significant difference in the laboratory indexes between the two groups before treatment, and all participants achieved complete remission after surgery. As of December
2019 , there were 34 cases of intrahepatic recurrence and 25 cases of distant metastasis in control group, there were 24 cases of intrahepatic recurrences and 18 cases of distant metastases in the rhAd5 group. The incidence of recurrence and metastasis in the rhAd5 group were significantly lower than those in the control group. This result suggests that adjuvant chemotherapy combined with $\operatorname{rhAd} 5$ on the basis of FOLFOX can significantly inhibit the recurrence of HCC after surgery.

In recent years, the use of oncolytic viruses to treat malignant tumors is a new method in cancer treatment. Some oncolytic viruses are used in the treatment of HCC, especially adenovirus, which can improve the prognosis of patients. Currently, rhAd5 is used for the treatment of advanced HCC (23). Compared with TACE alone, rhAd5 given through TACE can significantly improve OS and PFS of HCC patients with unresectable tumors (18). However, 
A

Fatigue

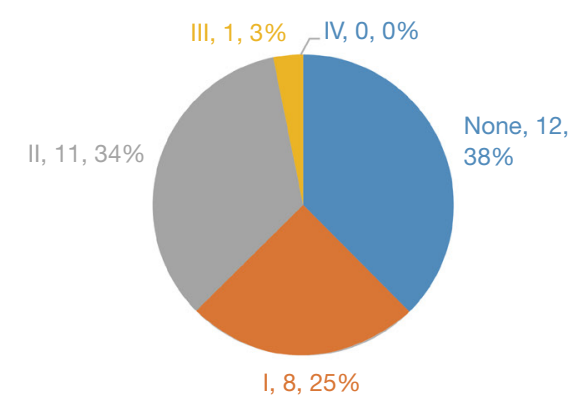

D

Bone marrow suppression

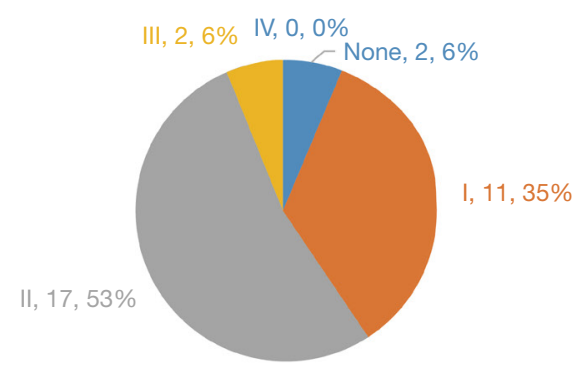

G

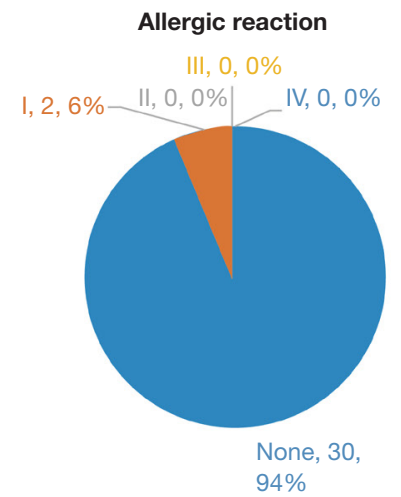

B

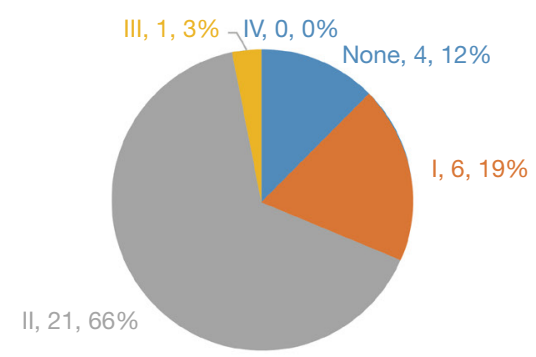

E

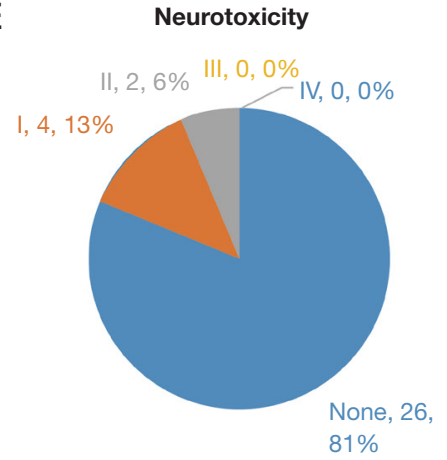

C Diarrhea

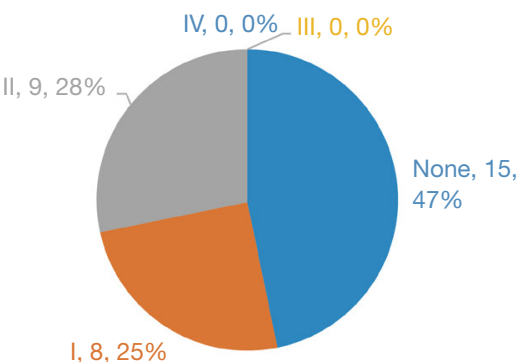

F

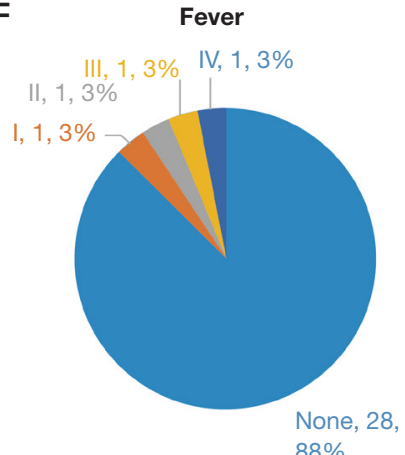

rhAd5 group

Figure 3 Occurrence of adverse reactions of the rhAd5 group.

there is no report about the use of rhAd5 for HCC after surgery. Some studies have shown that oncolytic viruses can be used after surgery and have positive effects of reducing recurrence. Ahmed et al. (24) synthesized a vaccinia virus (with deletion of both thymidine kinase and N1L genes), and it was found that it can inhibit the recurrence of postoperative solid tumors in vivo. A retrospective study has shown that the oncolytic non-pathogenic virus Rigvir can significantly reduce the recurrence of melanoma patients undergoing surgery and effectively prolong OS (25). Tai used oncolytic viruses to intervene during the perioperative period, and the results showed that it not only inhibits tumor metastasis, but also has the ability to regulate the body's immunity (26). These findings suggest that oncolytic viruses can alleviate tumor metastasis after surgery.

For chemotherapy, drug safety and adverse reactions are the two of the most noteworthy issues. In this study, we paid close attention to adverse reactions during treatment and follow-up, including clinical symptoms, liver function, kidney function, and blood routine indicators. It was shown that the use of rhAd5 after surgery has no significant effects on the above indexes. There was no statistically significant difference in the indicators and the occurrence of adverse reactions between the rhAd5 group and the control group. It is suggested that rhAd5 causes less additional harm to HCC patients. The rhAd5 virus is a modified oncolytic 
A

Fatigue

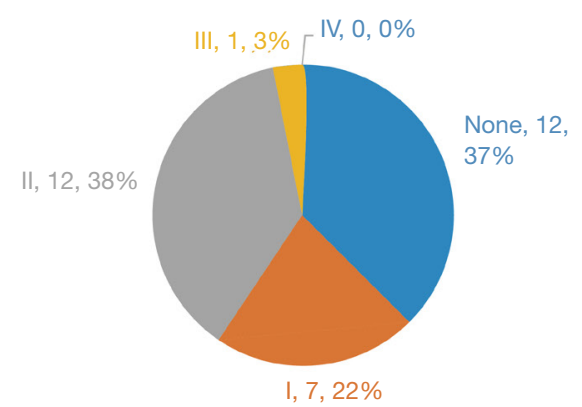

D

Bone marrow suppression

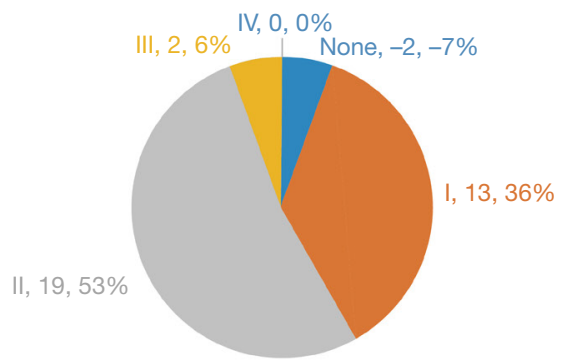

G
B

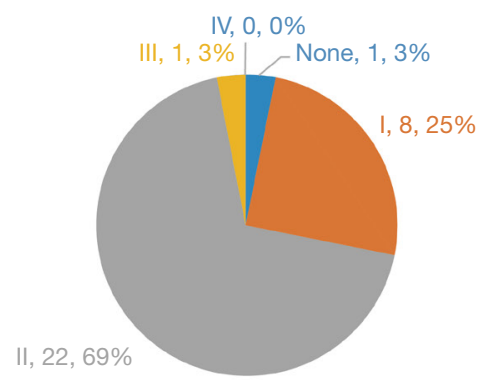

$\mathrm{E}$

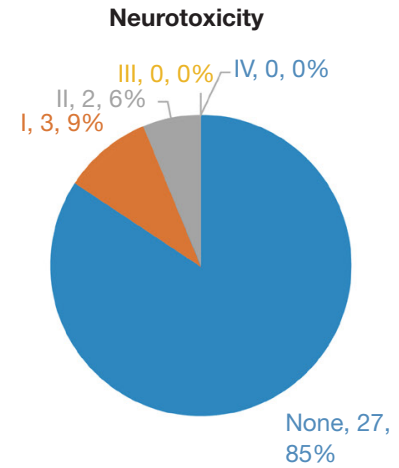

C

Diarrhea

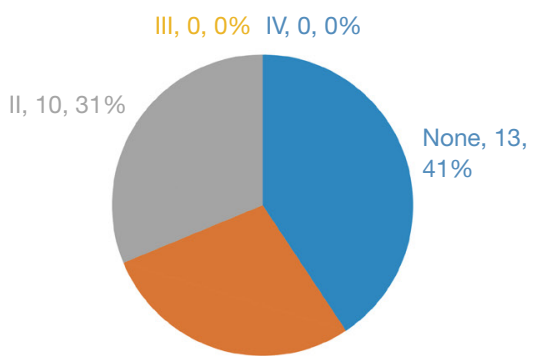

I, $9,28 \%$

$\mathrm{F}$

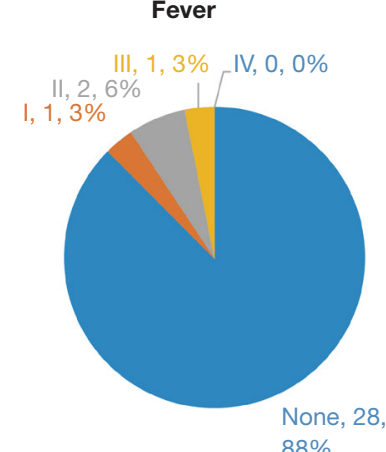

Contorl group

Figure 4 Occurrence of adverse reactions of the control group.

Table 2 Comparison of the laboratory indicators between the two groups

\begin{tabular}{lccccccc}
\hline Group & ALT peak & AST peak & TBIL peak & DBIL peak & WBC peak & HB valley & PLT valley \\
\hline rhAd5 group & $38.52 \pm 24.41$ & $38.3 \pm 20.97$ & $10.45 \pm 3.92$ & $2.89 \pm 1.53$ & $5.89 \pm 1.83$ & $116.97 \pm 15.62$ & $139.97 \pm 58.79$ \\
Control & $38.11 \pm 22.46$ & $35.14 \pm 17.82$ & $11.84 \pm 5.28$ & $3.21 \pm 2.52$ & $6.23 \pm 2.63$ & $121.64 \pm 15.35$ & $147.32 \pm 73.24$ \\
\hline
\end{tabular}

ALT, alanine transaminase; AST, aspartate transaminase; TBIL, total bilirubin; DBIL, direct bilirubin; HB, hemoglobin; PLT, platelet.

virus in which part of the E1B (E1B-5 $5 \mathrm{kD})$ gene has been deleted, and its replication ability in normal cells is suppressed, improving safety (27-29). This suggests that rhAd5 may target the lysis of residual HCC cells, thereby reducing the recurrence and metastasis after HCC.
In summary, the combination of FOLFOX and rhAd5 after surgery can significantly inhibit the occurrence of metastasis and recurrence of tumor in HCC patients, improve PFS, and have certain safety. It is still necessary to expand the sample size and extend the follow-up time, to 
further compare the impact of rhAd5 on prognosis and OS of HCC patients.

\section{Acknowledgments}

Funding: None.

\section{Footnote}

Reporting Checklist: The authors have completed the STROBE reporting checklist. Available at https://dx.doi. org/10.21037/jgo-21-792

Data Sharing Statement: Available at https://dx.doi. org/10.21037/jgo-21-792

Conflicts of Interest: All authors have completed the ICMJE uniform disclosure form (available at https://dx.doi. org/10.21037/jgo-21-792). The authors have no conflicts of interest to declare.

Ethical Statement: The authors are accountable for all aspects of the work in ensuring that questions related to the accuracy or integrity of any part of the work are appropriately investigated and resolved. All participants provided informed consent. This study was approved by the Ethics Review Committee of The Second Affiliated Hospital of Army Medical University (No.: CQ-012201434532) and was conducted in accordance with the Declaration of Helsinki (as revised in 2013).

Open Access Statement: This is an Open Access article distributed in accordance with the Creative Commons Attribution-NonCommercial-NoDerivs 4.0 International License (CC BY-NC-ND 4.0), which permits the noncommercial replication and distribution of the article with the strict proviso that no changes or edits are made and the original work is properly cited (including links to both the formal publication through the relevant DOI and the license). See: https://creativecommons.org/licenses/by-nc-nd/4.0/.

\section{References}

1. Bray F, Ferlay J, Soerjomataram I, et al. Global cancer statistics 2018: GLOBOCAN estimates of incidence and mortality worldwide for 36 cancers in 185 countries. CA Cancer J Clin 2018;68:394-424.

2. Zhu ZX, Huang JW, Liao MH, et al. Treatment strategy for hepatocellular carcinoma in China: radiofrequency ablation versus liver resection. Jpn J Clin Oncol 2016;46:1075-80.

3. Al-Judaibi B, Dokus MK. Surgical resection vs radiofrequency ablation in older adults with early stage hepatocellular carcinoma: Where do we stand? Saudi J Gastroenterol 2018;24:309-10.

4. Kondo H, Tomimaru Y, Iwazawa T, et al. Pulmonary Metastases of Hepatocellular Carcinoma Treated with Repeated Pulmonary Resection-A Case Report. Gan To Kagaku Ryoho 2019;46:2527-9.

5. Zhang YM, Zhou ZT, Liu GM. Factors predicting early recurrence after surgical resection of hepatocellular carcinoma. J Hepatol 2019;70:571-2.

6. Tsilimigras DI, Pawlik TM. ASO Author Reflections: Recurrence Patterns and Outcomes After Resection of Hepatocellular Carcinoma Within and Beyond the Barcelona Clinic Liver Cancer Criteria. Ann Surg Oncol 2020;27:2332-3.

7. Fukuda S, Okuda K, Imamura M, et al. Surgical resection combined with chemotherapy for advanced hepatocellular carcinoma with tumor thrombus: report of 19 cases. Surgery 2002;131:300-10.

8. Tamesa T, Mori N, Okada T, et al. A case of Vp4 hepatocellular carcinoma treated with surgical resection and continuous intrahepatic artery infusion chemotherapy of low-dose cisplatin and 5-fluorouracil. Gan To Kagaku Ryoho 2003;30:1769-72.

9. Jiang JM, Ohri N, Tang J, et al. Centers with more therapeutic modalities are associated with improved outcomes for patients with hepatocellular carcinoma. J Gastrointest Oncol 2019;10:546-53.

10. Harada K, Okamoto W, Mimaki S, et al. Comparative sequence analysis of patient-matched primary colorectal cancer, metastatic, and recurrent metastatic tumors after adjuvant FOLFOX chemotherapy. BMC Cancer 2019;19:255.

11. Niemann J, Kühnel F. Oncolytic viruses: adenoviruses. Virus Genes 2017;53:700-6.

12. Tilgase A, Grīne L, Blāķe I, et al. Effect of oncolytic ECHO-7 virus strain Rigvir on uveal melanoma cell lines. BMC Res Notes 2020;13:222.

13. Samson A, Scott KJ, Taggart D, et al. Intravenous delivery of oncolytic reovirus to brain tumor patients immunologically primes for subsequent checkpoint blockade. Sci Transl Med 2018;10:eaam7577.

14. Xiao J, Zhou J, Fu M, et al. Efficacy of recombinant human adenovirus-p 53 combined with chemotherapy for 
locally advanced cervical cancer: A clinical trial. Oncol Lett 2017;13:3676-80.

15. Sun YC, Li TT, Wang YL, et al. Detection of neutralizing antibody to human adenovirus type 5 in marmosets. Nan Fang Yi Ke Da Xue Xue Bao 2016;36:582-7.

16. Steffen T, Hassert M, Hoft SG, et al. Immunogenicity and Efficacy of a Recombinant Human Adenovirus Type 5 Vaccine against Zika Virus. Vaccines (Basel) 2020;8:170.

17. Dong J, Li W, Dong A, et al. Gene therapy for unresectable hepatocellular carcinoma using recombinant human adenovirus type 5. Med Oncol 2014;31:95.

18. Lin XJ, Li QJ, Lao XM, et al. Transarterial injection of recombinant human type-5 adenovirus H101 in combination with transarterial chemoembolization (TACE) improves overall and progressive-free survival in unresectable hepatocellular carcinoma (HCC). BMC Cancer 2015;15:707.

19. Ikegami T, Yoshizumi T, Kawasaki J, et al. Surgical Resection for Lymph Node Metastasis After Liver Transplantation for Hepatocellular Carcinoma. Anticancer Res 2017;37:891-5.

20. Tabrizian P, Jibara G, Shrager B, et al. Recurrence of hepatocellular cancer after resection: patterns, treatments, and prognosis. Ann Surg 2015;261:947-55.

21. Galle PR, Tovoli F, Foerster F, et al. The treatment of intermediate stage tumours beyond TACE: From surgery to systemic therapy. J Hepatol 2017;67:173-83.

22. Lee JB, Kim HS, Jung I, et al. Upfront radical surgery with total mesorectal excision followed by adjuvant FOLFOX chemotherapy for locally advanced rectal cancer (TME-FOLFOX): an open-label, multicenter, phase II randomized controlled trial. Trials 2020;21:320.

23. He CB, Lin XJ. Inflammation scores predict the survival of patients with hepatocellular carcinoma who were treated with transarterial chemoembolization and recombinant human type-5 adenovirus H101. PLoS One 2017;12:e0174769.

24. Ahmed J, Chard LS, Yuan M, et al. A new oncolytic V accinia virus augments antitumor immune responses to prevent tumor recurrence and metastasis after surgery. J Immunother Cancer 2020;8:e00415.

25. Doniņa S, Strēle I, Proboka G, et al. Adapted ECHO7 virus Rigvir immunotherapy (oncolytic virotherapy) prolongs survival in melanoma patients after surgical excision of the tumour in a retrospective study. Melanoma Res 2015;25:421-6.

26. Tai LH, Auer R. Attacking Postoperative Metastases using Perioperative Oncolytic Viruses and Viral Vaccines. Front Oncol 2014;4:217.

27. Heise C, Sampson-Johannes A, Williams A, et al. ONYX015, an E1B gene-attenuated adenovirus, causes tumorspecific cytolysis and antitumoral efficacy that can be augmented by standard chemotherapeutic agents. Nat Med 1997;3:639-45.

28. Hay JG, Shapiro N, Sauthoff H, et al. Targeting the replication of adenoviral gene therapy vectors to lung cancer cells: the importance of the adenoviral E1b-55kD gene. Hum Gene Ther 1999;10:579-90.

29. Yu W, Fang H. Clinical trials with oncolytic adenovirus in China. Curr Cancer Drug Targets 2007;7:141-8.

(English Language Editor: J. Jones)
Cite this article as: Wu K, You N, Zheng L. Effects of recombinant human adenovirus type 5 combined with transarterial chemoembolization on postoperative metastasis and recurrence of hepatocellular carcinoma patients. J Gastrointest Oncol 2021;12(6):2999-3007. doi: 10.21037/jgo-21-792 